\title{
VLBA SiO observations of bright O-rich AGB stars
}

\author{
W. D. Cotton ${ }^{1}$, G. Perrin ${ }^{2}$, and B. Lopez ${ }^{3}$ \\ 1 National Radio Astronomy Observatory ${ }^{\star}, 520$ Edgemont Road, Charlottesville, VA 22903-2475, USA \\ e-mail: bcotton@nrao . edu \\ 2 Observatoire de Paris, LESIA, UMR 8109, 92190 Meudon, France \\ 3 Observatoire de la Côte d'Azur, Nice, France
}

Received 8 May 2007 / Accepted 22 October 2007

\section{ABSTRACT}

\begin{abstract}
We present the results of observations of the $\mathrm{SiO}$ masers at $7 \mathrm{~mm}$ wavelength in several oxygen rich Asymptotic Giant Branch (AGB) stars. These observations were coordinated with observations using the ESO VLTI/MIDI interferometer at $10 \mu \mathrm{m}$ wavelength. These stars are $o$ Ceti (Mira), U Herculis (U Her), OH26.5+0.6, R Leonis (R Leo), and W Hydrae (W Hya). Both total intensity and linear polarizations are imaged. Linear radial features seen in $o$ Ceti may be associated with an X-ray flare seen a year earlier by Karovska et al. (2005). Similar linear features are also seen in R Leo, U Her and OH26.5+0.6. These features typically show systematic radial velocity variations and a tendency for the linear polarization to be perpendicular to the direction of elongation, suggesting that magnetic fields are dynamically important. A wide radial extent of the maser rings observed in $\mathrm{U}$ Herculis and $\mathrm{OH} 26.5+0.6$ is interpreted as meaning that the outer boundaries of the masing regions in these stars are not truncated by the condensation of SiO onto dust grains.
\end{abstract}

Key words. stars: atmospheres - stars: AGB and post-AGB - stars: variables: general - radio lines: stars - masers

\section{Introduction}

Asymptotic Giant Branch (AGB) stars are stars of a few solar masses and have reached the end of their lives; the inner core of the star has collapsed and the outer envelope has become very extended and is in the process of losing its mass to the interstellar medium through pulsational instabilities. In the cool, extended envelopes of these stars, molecules form and condense into dust. In oxygen rich stars, molecular masers appear, especially $\mathrm{SiO}$, $\mathrm{H}_{2} \mathrm{O}$ and $\mathrm{OH}$ at respectively increasing distance from the photosphere (Reid \& Moran 1981). The SiO masers occur within a few stellar radii of the stellar surface between the hot molecular inner envelope and the cooler region at $3-5 R_{\star}$ where the (silicate) dust forms; see Reid \& Menten (1997), Danchi et al. (1994). The detailed mechanics driving mass loss is not well understood; nor is the reason that many planetary nebulae are very asymmetric whereas their AGB progenitors appear circularly symmetric.

Circumstellar $\mathrm{SiO}$ masers tend to occur in clumpy, partial rings centered on the central star (Diamond et al. 1994). A long path through relatively constant (radial) velocity gas is needed for the masers to develop the necessary gain to be visible. This condition is most often met for lines of sight tangent to the masing layer in regions of relatively low turbulence. Turbulence will reduce the path length at a given velocity limiting the gain, hence brightness, of the corresponding maser spot. The resulting maser spots tend to appear in a partial ring. Thus, these masers become markers for the layer in which the physical conditions necessary for the masers exist and tracers of the dynamics of these regions. Humphreys et al. (1996) and Humphreys et al. (2002) discuss the dynamics of circumstellar $\mathrm{SiO}$ masers.

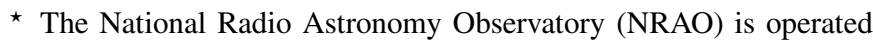
by Associated Universities Inc., under cooperative agreement with the National Science Foundation.
High resolution observations of the $\mathrm{SiO}$ masers in this dynamically significant region provide a powerful probe. The frequent monitoring of the $\mathrm{SiO}$ masers in TX Cam by Diamond \& Kemball (2003) show complex motions of the masing gas; both outward and inward motions are detected.

The polarization of circumstellar masers is a valuable probe of magnetic fields in circumstellar envelopes. The complex theory of maser polarization, and particularly that of the $\mathrm{SiO}$ masers, is described in a number of publications (e.g. Deguchi \& Watson 1990; Nedoluha \& Watson 1990; Elitzur 1991; Nedoluha \& Watson 1994; Elitzur 1996). Observations of TX Cam by Kemball \& Diamond (1997) revealed tangentially ordered polarization vectors indicating a radial magnetic field direction and circular polarization indicating a magnetic field strength of several Gauss. Cotton et al. (2006) also found some evidence for ordered fields in a number of stars as well as evidence of a significant dynamical role of magnetic fields in a number of stellar $\mathrm{SiO}$ masers. The influence of magnetic fields is illustrated by the recent detection of a magnetically collimated jet around an evolved star (Vlemmings et al. 2006).

One of the difficulties in understanding these stars is that of determining the apparent photospheric size. Ireland et al. (2004) have used the aperture masking interferometry technique to measure the sizes of a number of AGB stars in the red through near IR. The sizes of AGB stars all increase systematically towards the blue and were larger than expected at all wavelengths. The authors speculated that this was due to scattering by dust in the inner envelope. Ireland \& Scholz (2006) calculate various models of dust formation and conclude that for a number of cases, the inner diameter of the dust shell would be 2-3 times that of the photosphere. Monnier et al. (2004) used a combination of aperture masking on Keck with interferometric measurements using IOTA at $2.2 \mu \mathrm{m}$ to image a number of stars with dust shells. They 
determined that the shells in a number of stars, including Miras were clumped.

Perrin et al. (2004) observed a number of Mira variables between 2.2 and $3.6 \mu \mathrm{m}$ and derived large variations of the apparent size with wavelength. They interpreted the variation in size as being due to a molecular shell containing $\mathrm{H}_{2} \mathrm{O}$ and a wavelength dependent opacity.

Ohnaka et al. (2005) observed the Mira variable RR Sco at 2.2 and $10 \mu \mathrm{m}$ using the VLTI and modeled the star with a photosphere surrounded by an optically thick envelope of $\mathrm{H}_{2} \mathrm{O}$ and $\mathrm{SiO} 2.3$ times the photospheric size and surrounded by an optically thin dust shell with an inner diameter of 7-8 times the photospheric size. $11 \mu \mathrm{m}$ observations of Danchi et al. (1994) also found considerable variations in the ratio of the size of the inner dust shell to the size of the photosphere. However, Cotton et al. (2004) found a fairly small scatter of the ratio of the $\mathrm{SiO}$ maser ring diameter to the apparent size at $2.2 \mu \mathrm{m}$ around a value of approximately 2 . The relation of the $\mathrm{SiO}$ maser ring diameter to the inner size of the dust shell is unclear.

In this paper we report the VLBA observational part of a coordinated set of observations using the VLBA and the ESO VLTI/MIDI interferometers. The VLTI/MIDI instrument works in the mid infrared at around $10 \mu \mathrm{m}$ and is very sensitive to the region of dust formation. The $\mathrm{SiO}$ maser observations serve as a probe of the physical conditions and dynamics of the stellar envelope just interior to the region of dust formation. Initial VLTI/MIDI results are given in Menut et al. (2004), Chesneau et al. (2005) and Ohnaka et al. (2005). Further VLTI/MIDI results and a joint interpretation will be reported in subsequent publications.

\section{Observations and data reduction}

The new observations were obtained with the NRAO Very Long Baseline Array (VLBA) during a $20 \mathrm{~h}$ session on 5 September 2004. Two $4 \mathrm{MHz}$ wide channels in each rightand left-hand circular polarization were recorded at 42.820587 and $43.122027 \mathrm{GHz}$ to cover the $v=2, J=1-0$ and $v=1, J=$ $1-0$ transitions of $\mathrm{SiO}$. Two bit sampling was used in the recording. The correlations resulted in 128 channels in each of the combinations of right- and left-circular polarization for each transition. A strong, nearby continuum source was observed before each star to serve as delay, bandpass and polarization calibrator. The observations are summarized in Table 1 which lists the stars, calibrators, and central velocities. The calibrators are all quasars with milliarcsecond accuracy positions; the Hipparcos (ESA 1997) positions of the stars (except OH26.5+0.6), evaluated at the epoch of the observations, were used. Note: the center velocities were picked to center the spectra in the observing band and are not necessarily the stellar systemic velocities.

Calibration and processing follows the procedure described in Cotton et al. (2004) and Cotton et al. (2006) except as noted below. Calibration was done in the NRAO AIPS package and imaging and subsequent processing in the Obit package (http://www.cv.nrao.edu/ bcotton/Obit.html). Linear polarization calibration was based on monitoring of the continuum calibrators from the VLA and, in general, the calibration of polarization angle should be better than 10 degrees. An error in the calibration of the polarization angle will be common to all images shown here. Velocity images are derived from a flux density weighted average velocity of pixels with significant emission.
Table 1. AGB stars observed.

\begin{tabular}{lcrrrr}
\hline \hline Star & Calibrator & $\begin{array}{r}\text { Vel A } \\
\left(\mathrm{km} \mathrm{s}^{-1}\right)\end{array}$ & $\begin{array}{r}\text { Vel B }^{2} \\
\left(\mathrm{~km} \mathrm{~s}^{-1}\right)\end{array}$ & $\begin{array}{r}\text { Distance }^{3} \\
\mathrm{pc}\end{array}$ & Phase $^{4}$ \\
\hline$o$ Ceti & $\mathrm{J} 0217+0144$ & 48.0 & 45.0 & $128 \pm 18$ & 0.35 \\
R Leonis & $\mathrm{J} 0854+2006$ & 2.0 & -1.0 & $101 \pm 20$ & 0.00 \\
W Hydrae & $\mathrm{J} 1337-1257$ & 41.7 & 41.7 & $115 \pm 14$ & 0.65 \\
U Herculis & $\mathrm{J} 1613+3412$ & -14.0 & -16.0 & $610 \pm 490$ & 0.34 \\
OH26.5+0.6 & $\mathrm{J} 1751+0939$ & 29.0 & 29.0 & & 0.34 \\
\hline
\end{tabular}

${ }^{1} v=2, J=1-0$ transition of $\mathrm{SiO}$ at $42.8 \mathrm{GHz} ;{ }^{2} v=1, J=1-0$ transition of $\mathrm{SiO}$ at $43.1 \mathrm{GHz} ;{ }^{3}$ Distance from Hipparcos (ESA 1997) parallax; ${ }^{4}$ Pulsation phase.

\section{Results}

The integrated total intensity and velocity images for each transition with superposed polarization "E" vectors are shown in Figs. 1-5.

\subsection{Ring diameter}

The diameters and widths of the $\mathrm{SiO}$ maser rings were determined from a moment analysis of compressed (2D) Stokes I images in both transitions using a direct parameter search which minimized the sum of the ring widths. The independent ring diameters and widths were determined jointly for the two transitions but with a common center. This procedure, especially the estimated ring widths may be adversely affected by residual errors in the alignment of the images in the two transitions. The distribution of emission in $\mathrm{OH} 26.5+0.6$ was sufficiently asymmetric that the center was not well fitted so this was estimated "by eye". The fitted ring diameters and width for each star and transition are shown in Table 2.

\subsection{Polarization}

The vectors in Figs. 1-5 are proportional to the linearly polarized intensity, and their orientation defines the plane of the electric field. The relationship between the orientation of linear polarization and the magnetic field is relatively complex (see discussion in Cotton et al. (2006)) but under many circumstances, the emitted linear polarization is perpendicular to the magnetic field.

The magnetic field strength can be measured directly from Zeeman splitting of the maser lines in Stokes $V$ measurements. Unfortunately, processing the data from these observations pushed the limits of the capacity of the VLBA correlator and it was not possible to obtain sufficient spectral resolution to make meaningful measurements of the Zeeman splitting.

\section{3. o Ceti}

The images of the masers in $o$ Ceti seen in Fig. 1 show an irregular shape approximately 74 mas in diameter at pulsation phase 0.35 . This is consistent with results from several epochs reported by Cotton et al. (2004) and Cotton et al. (2006). Only portions of a ring structure are seen. The polarized regions shown in Fig. 1 are largely associated with linear, radial features and the polarization is generally perpendicular to the features suggesting a parallel magnetic field. 

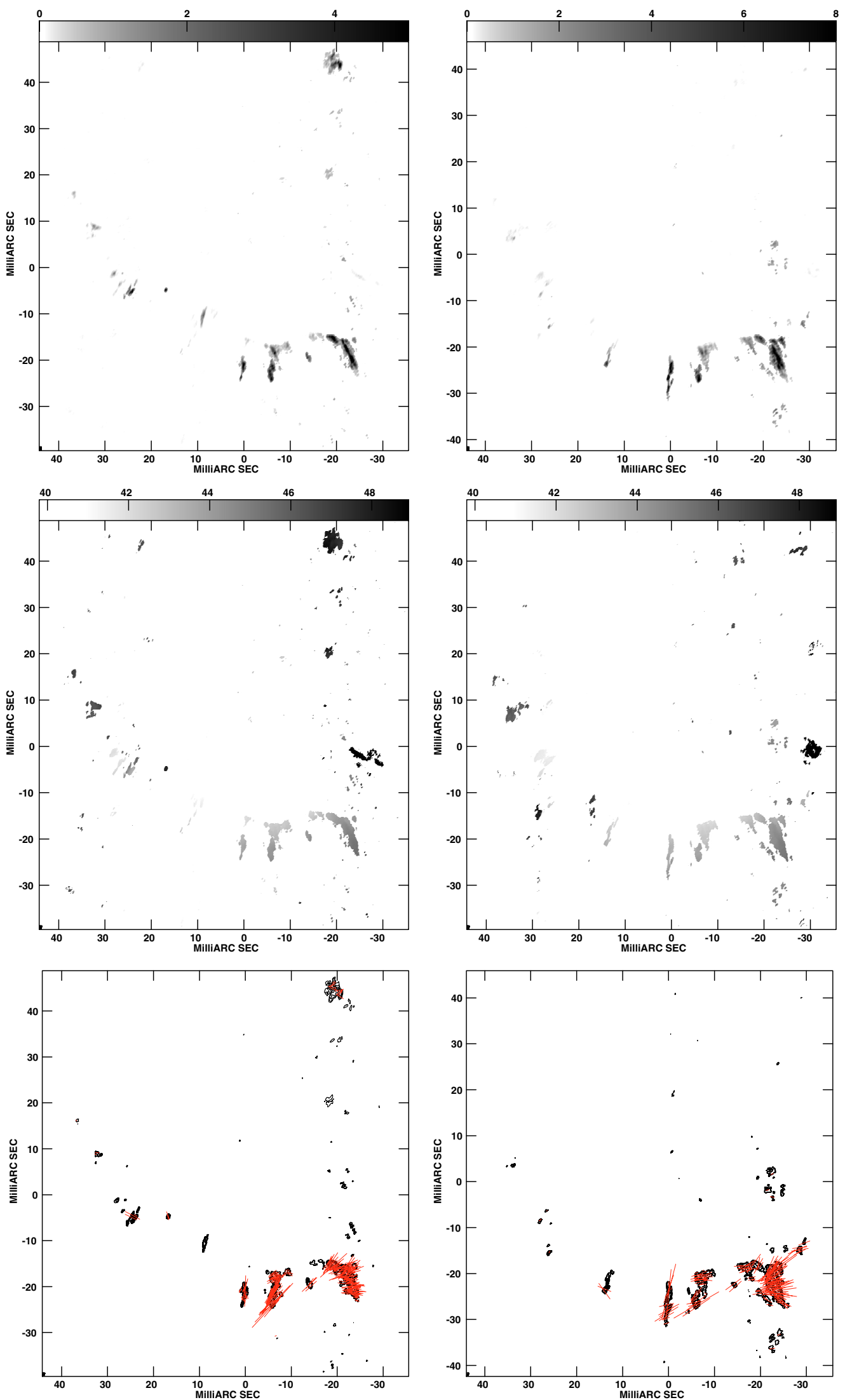

Fig. 1. $o$ Ceti (Mira). The left column is the $v=2, J=1-0$ transition of $\mathrm{SiO}$ at $42.8 \mathrm{GHz}$, the right column is the $v=1, J=1-0$ transition of $\mathrm{SiO}$ at $43.1 \mathrm{GHz}$. The top plots are the total intensity as a reversed gray-scale, the middle plots are velocity averages of pixels with significant emission, and the bottom plots are total intensity contours (spaced by powers of two) with polarization "E" vectors over-plotted in red. 

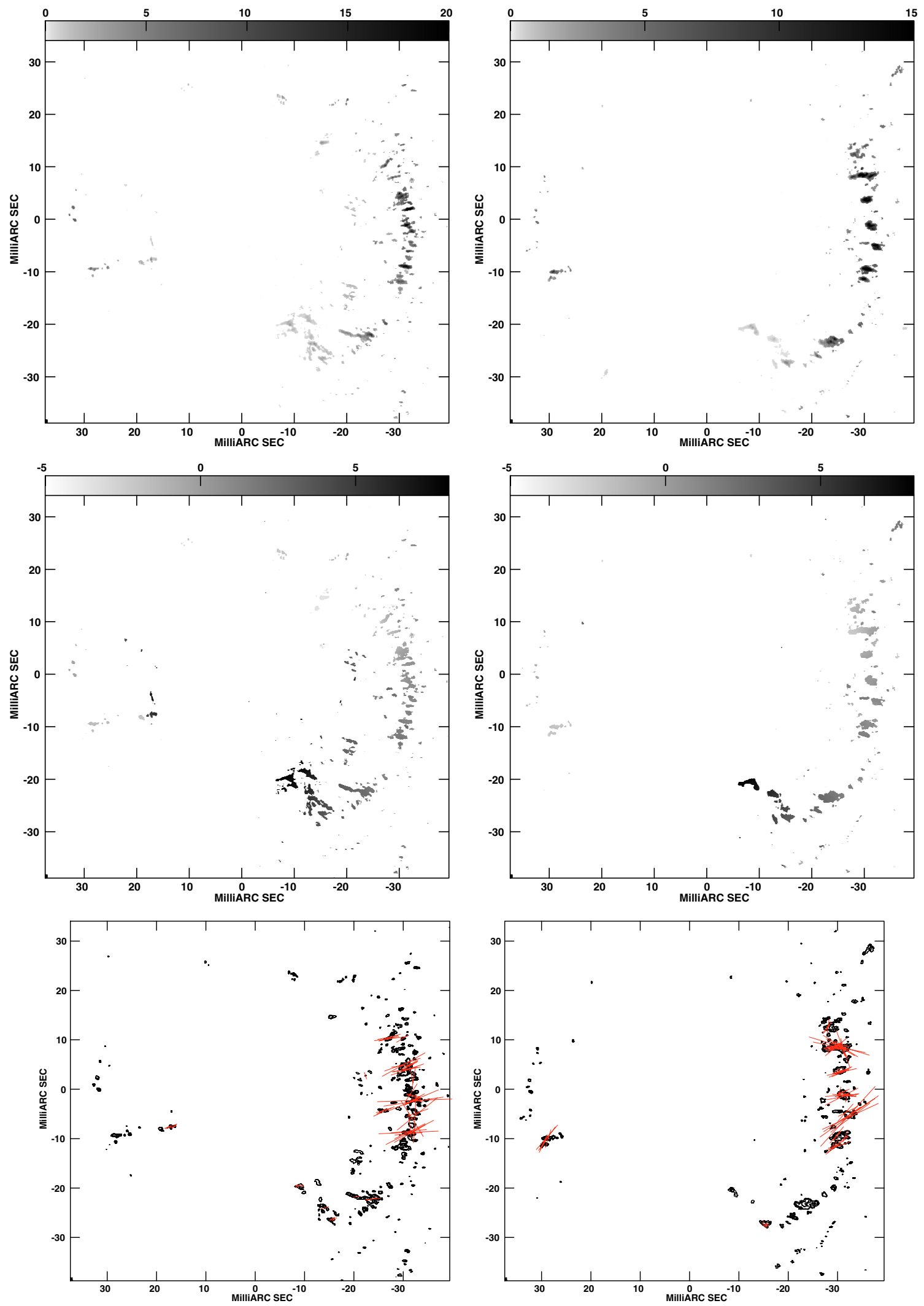

Fig. 2. R Leonis. The left column is the $v=2, J=1-0$ transition of $\mathrm{SiO}$ at $42.8 \mathrm{GHz}$, the right column is the $v=1, J=1-0$ transition of $\mathrm{SiO}$ at 43.1 GHz. The top plots are the total intensity as a reversed gray-scale, the middle plots are velocity averages of pixels with significant emission, and the bottom plots are total intensity contours (spaced by powers of two) with polarization "E" vectors over-plotted in red.

\subsection{R Leonis}

The masers in R Leonis seen in Fig. 2 show a partial ring approximately 62 mas in diameter at pulsation phase 0.00 . This is consistent with previous results over a range of phase. Cotton et al. (2004) also show partial rings, but mostly on the northern and eastern sides whereas in Fig. 2 a well developed structure 

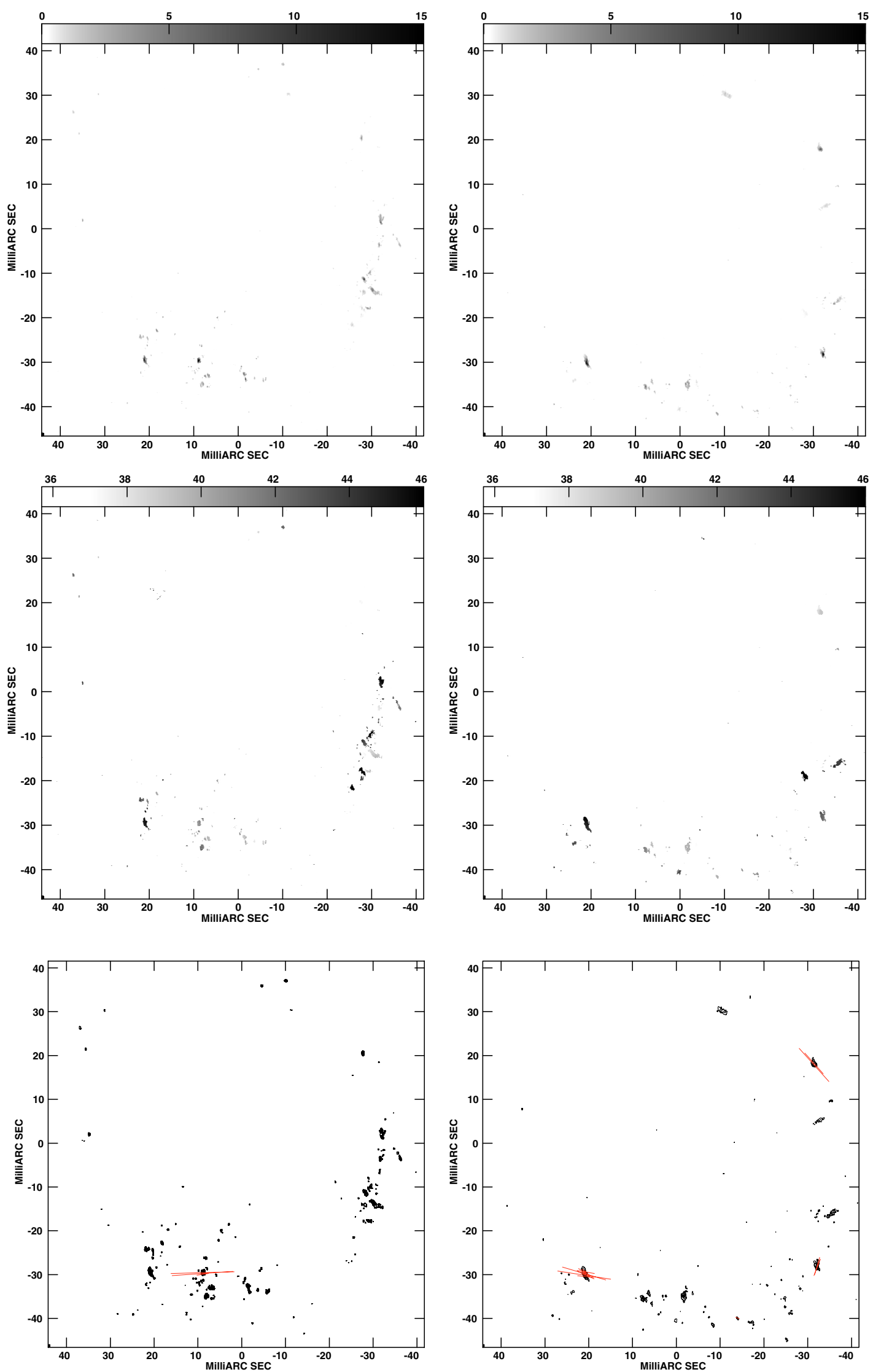

Fig. 3. W Hydrae. The left column is the $v=2, J=1-0$ transition of $\mathrm{SiO}$ at $42.8 \mathrm{GHz}$, the right column is the $v=1, J=1-0$ transition of $\mathrm{SiO}$ at 43.1 GHz. The top plots are the total intensity as a reversed gray-scale, the middle plots are velocity averages of pixels with significant emission, and the bottom plots are total intensity contours (spaced by powers of two) with polarization "E" vectors over-plotted in red. 

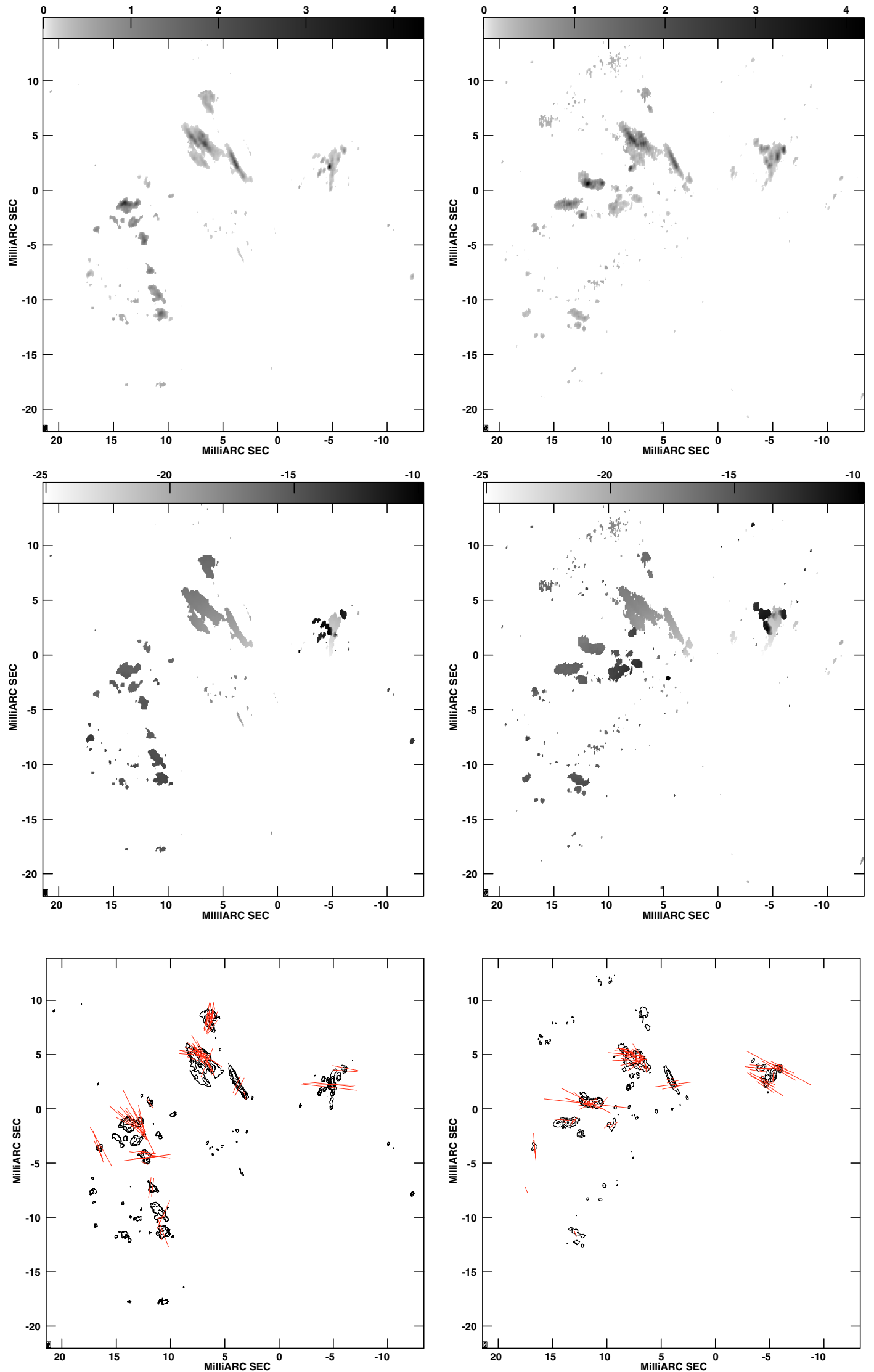

Fig. 4. U Herculis. The left column is the $v=2, J=1-0$ transition of $\mathrm{SiO}$ at $42.8 \mathrm{GHz}$, the right column is the $v=1, J=1-0$ transition of $\mathrm{SiO}$ at 43.1 GHz. The top plots are the total intensity as a reversed gray-scale, the middle plots are velocity averages of pixels with significant emission, and the bottom plots are total intensity contours (spaced by powers of two) with polarization "E" vectors over-plotted in red. 

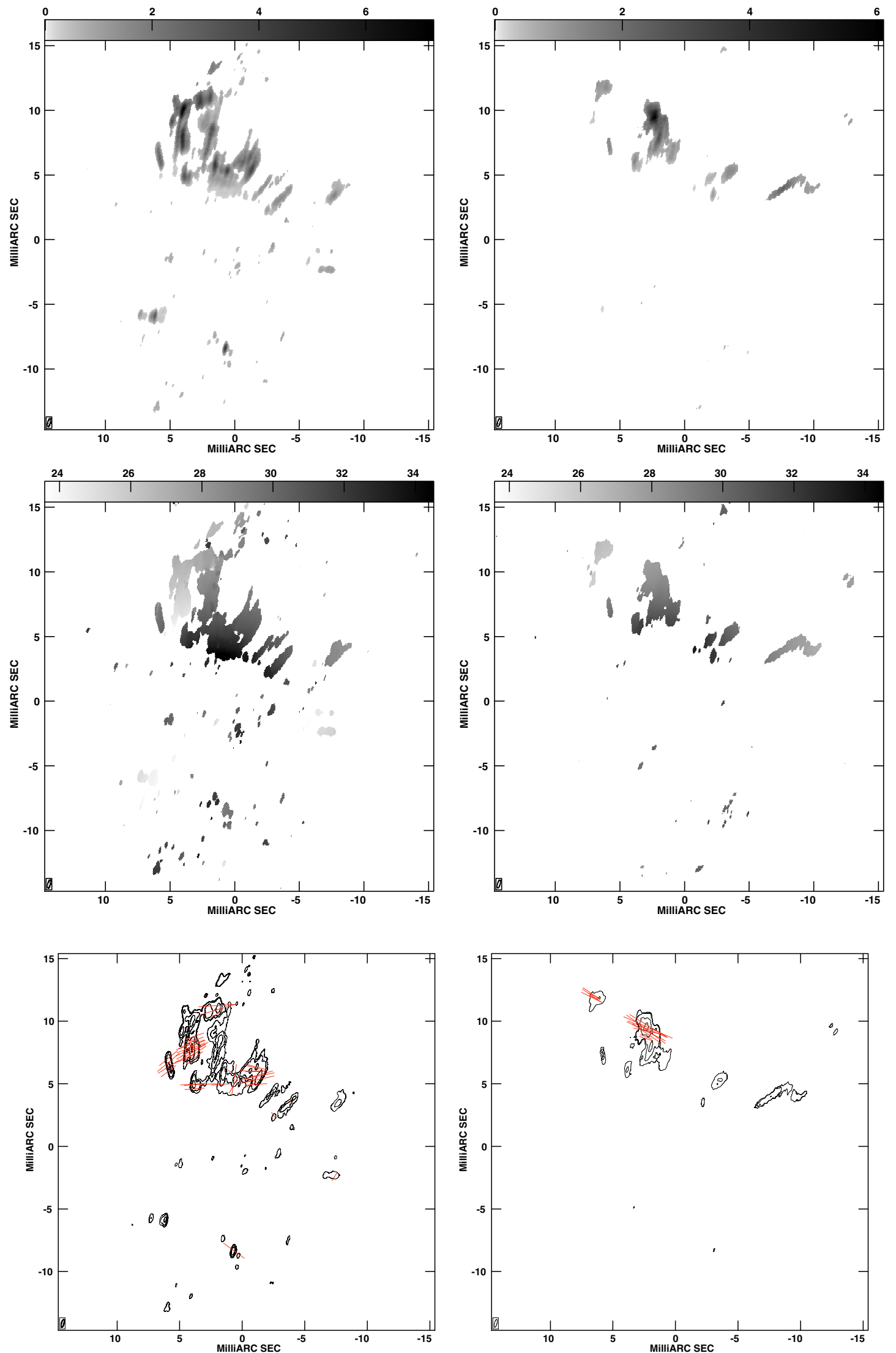

Fig. 5. $\mathrm{OH} 26.5+0.6$. The left column is the $v=2, J=1-0$ transition of $\mathrm{SiO}$ at $42.8 \mathrm{GHz}$, the right column is the $v=1, J=1-0$ transition of $\mathrm{SiO}$ at 43.1 GHz. The top plots are the total intensity as a reversed gray-scale, the middle plots are velocity averages of pixels with significant emission, and the bottom plots are total intensity contours (spaced by powers of two) with polarization "E" vectors over-plotted. 
Table 2. SiO ring diameters.

\begin{tabular}{|c|c|c|c|c|c|c|c|c|}
\hline Source & $\begin{array}{r}v=1^{1} \\
\operatorname{diam}(\mathrm{mas})\end{array}$ & width & $\begin{array}{r}v=2^{2} \\
\operatorname{diam}(\mathrm{mas})\end{array}$ & width & $\begin{array}{r}v=1 \\
\operatorname{diam}(\mathrm{AU})\end{array}$ & width & $\begin{array}{r}v=2 \\
\operatorname{diam}(\mathrm{AU})\end{array}$ & width \\
\hline$o$ Ceti & 71.1 & 2.6 & 68.6 & 2.3 & 9.1 & 0.3 & 8.8 & 0.3 \\
\hline R Leonis & 62.1 & 0.7 & 62.6 & 0.6 & 6.3 & 0.07 & 6.3 & 0.06 \\
\hline W Hydrae & 72.0 & 1.3 & 60.0 & 3.2 & 8.3 & 0.15 & 6.9 & 0.4 \\
\hline U Herculis & 22.9 & 1.1 & 22.0 & 1.4 & 14.0 & 0.7 & 13.4 & 0.9 \\
\hline $\mathrm{OH} 26.5+0.6^{3}$ & 22.1 & 0.3 & 19.4 & 2.2 & & & & \\
\hline
\end{tabular}

Notes: ${ }^{1} v=1, J=1-0$ transition of $\mathrm{SiO}$ at $43.1 \mathrm{GHz} ;{ }^{2} v=2, J=1-0$ transition of $\mathrm{SiO}$ at $42.8 \mathrm{GHz} ;{ }^{3}$ Center not fitted, no parallax available.
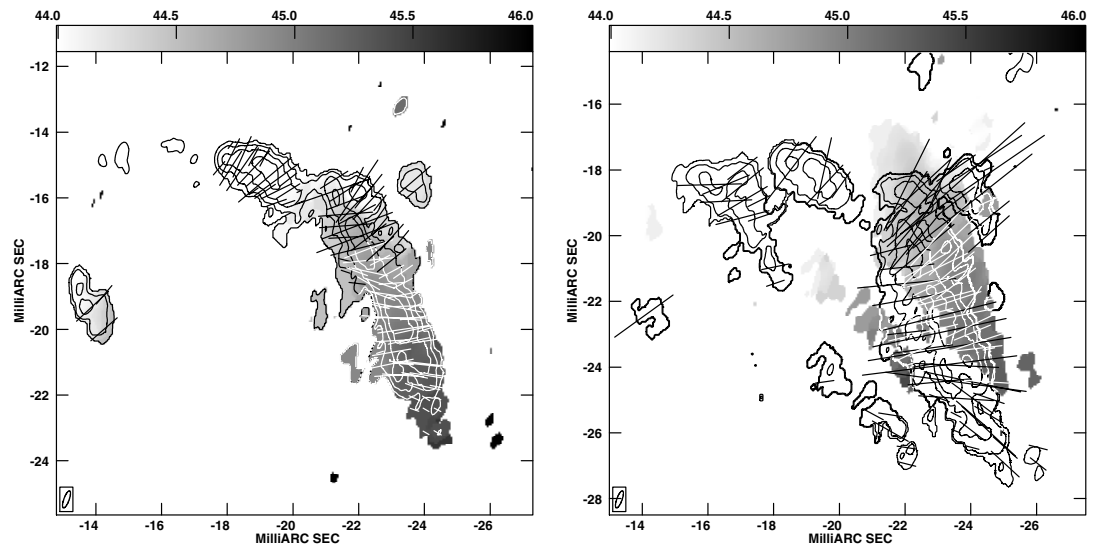

Fig. 6. $o$ Ceti. Closeup of a region in Fig. 1. Left is the $v=2, J=1-0$ transition of $\mathrm{SiO}$ at $42.8 \mathrm{GHz}$, on the right is the $v=1, J=1-0$ transition of $\mathrm{SiO}$ at $43.1 \mathrm{GHz}$. The gray scale is the average velocity as given by the scale-bar at the top of each plot, contours are total intensity with an interval of powers of two, and polarization "E" vectors are over-plotted. The center of the star is to the upper left. The resolution is shown in a box in the lower left corner.

is seen on the western side. The maser ring appears larger than was typical in the 2001 observations of Cotton et al. (2004). The polarization vectors shown in Fig. 2 are largely perpendicular to the ring suggesting a tangential field.

\subsection{W Hydrae}

The masers in W Hydrae (Fig. 3) form a symmetric partial ring to the south and west with a diameter of $\sim 70$ mas at pulsation phase 0.65 . This is substantially smaller than the $\sim 80$ mas diameter reported by Cotton et al. (2004) (phase 0.53). Only a few of the maser spots show significant linear polarization although the polarization angle is generally tangent to the ring suggesting a radial nature of the magnetic field.

\subsection{U Herculis}

The masers in $U$ Herculis shown in Fig. 4 exhibit an irregular shape approximately 20 mas in size at pulsation phase 0.34 . This is somewhat smaller that the typical size, $\sim 25$ mas, at multiple epochs in 2001 reported by Cotton et al. (2004) and with a shape far more irregular than the 2001 results. The polarization vectors shown in Fig. 4 are relatively chaotic although with a tendency to be orthogonal to linear features.

\section{7. $\mathrm{OH} 26.5+0.6$}

The masers in $\mathrm{OH} 26.5+0.6$ (see Fig. 5) largely consist of plumes to the north of a ring with a diameter of 13.7 mas defined by several bright spots. The polarized regions in Fig. 5 are mostly associated with the plumes to the north and show a weak tendency for the orientation to be orthogonal to structures.

\section{Jet-like features}

In a number of the maser images presented here and in Cotton et al. (2006) there are extended, linear features aligned with the direction to the photosphere. These frequently have similar morphologies in both the transitions observed with similar runs of velocity which vary systematically along their lengths. Since these features are near the masing ring, these velocities will be predominantly non-radial with respect to the star so do not represent simple acceleration of the masing material away from the star. The apparent alignment of the magnetic field with these features could be due either to the magnetic field being stretched along the features by the ionized component of the gas or the gas being constrained by its ionized component to move along field lines, depending on the relative energy density of the magnetic field. SiO maser observations (Herpin et al. 2003, 2006) and $\mathrm{H}_{2} \mathrm{O}$ maser observations (Vlemmings et al. 2005, 2006) suggest that magnetic fields are likely dynamically important in which case the maser jet-like features are following the magnetic field.

\section{1. o Ceti}

Jet-like features in $o$ Ceti have previously been discussed by Cotton et al. (2006) in which the radial velocities were furthest from systemic closest to the star and the polarization " $E$ " vectors perpendicular to the feature. A close up view of the linear features in Fig. 1 is given in Fig. 6. These features are at a very different orientation than the 2001 features but also show radial velocities which are furthest from systemic closest to the star and the polarization "E" vectors perpendicular to the features. 


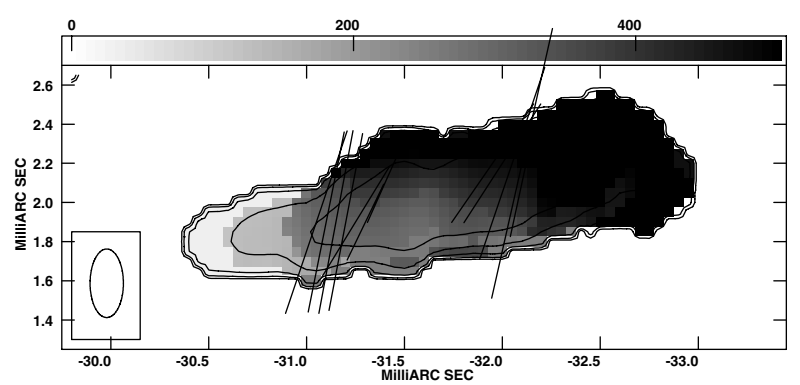

Fig. 7. R Leonis. Closeup of a region in Fig. 2 in the $v=2, J=1-0$ transition of $\mathrm{SiO}$ at $42.8 \mathrm{GHz}$. The gray scale is the average velocity as given by the scale-bar at the top, contours are total intensity with an interval of powers of two, and polarization "E" vectors are over-plotted. The center of the star is to the left. The resolution is shown in a box in the lower left corner.

\section{2. $R$ Leonis}

Figure 2 shows a number of linear features to the west of the star; the most prominent of these is shown in more detail in Fig. 7. In this case, the velocities closest to the star are closest to systemic. While the polarization from this feature is weak, the polarization vectors are more nearly perpendicular than parallel.

\subsection{U Herculis}

One of the more prominent regions of plumes to the north seen in Fig. 4 is shown in detail in Fig. 8. In this case the regions closest to the star are further from systemic than more distant regions. The linear polarization along the features shown in Fig. 8 show a mixture of orientation to the direction of the feature, parallel, perpendicular and oblique angles are seen. The narrowest feature has polarization "E" vectors perpendicular to the direction.

\section{4. $\mathrm{OH} 26.5+0.6$}

Details of the regions of maser plumes to the north in Fig. 5 are shown in Fig. 9. The velocities in these features show a complex pattern including the entire range of velocities seen in this star. The linear polarization in the better defined linear features is perpendicular to the feature.

\section{Discussion}

The general picture of the atmospheres of O-rich AGB stars is that the $\mathrm{SiO}$ maser appear just interior to the region in which the dust forms. Since the dust cannot condense until the gas temperature drops below the condensation temperature, the dust will form a shell around the star. A comparison of measured or modeled inner diameters of the circumstellar dust shells can test this picture. Previous IR measurements and the mean values from Table 2 are shown in Table 3 . Note, the $2.2 \mu$ m diameters include contributions from the molecular layer and are larger than the photosphere.

\section{1. o Ceti}

An X-ray outburst was reported in Mira A (the AGB component) on 6 Dec. 2003 by Karovska et al. (2005). It was postulated that this event was associated with a large mass ejection event and a dust ejection was predicted.

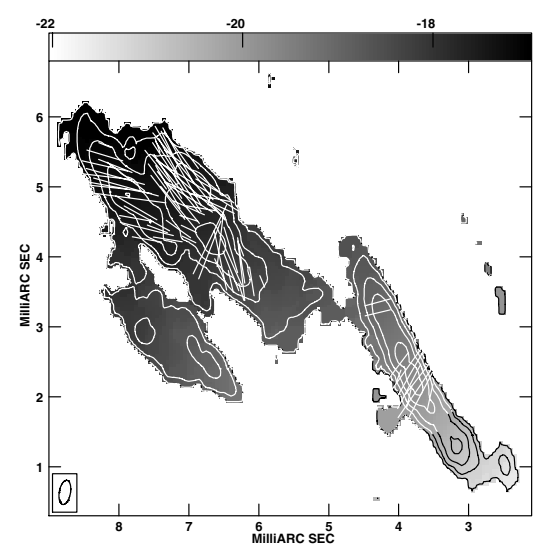

Fig. 8. U Herculis. Closeup of a region in Fig. 4 in the $v=2, J=$ $1-0$ transition of $\mathrm{SiO}$ at $42.8 \mathrm{GHz}$,. The gray scale is the average velocity as given by the scale-bar at the top, contours are total intensity with an interval of powers of two, and polarization "E" vectors are overplotted. The star center is to the lower right. The resolution is shown in a box in the lower left corner.

Table 3. IR diameters.

\begin{tabular}{lrrrr}
\hline \hline Source & $\begin{array}{r}2.2 \mu \mathrm{m} \\
\text { diam }(\mathrm{mas})\end{array}$ & $\begin{array}{r}\text { Photo } 11 \mu \mathrm{m} \\
\text { diam }(\mathrm{mas})\end{array}$ & $\begin{array}{r}\text { Dust } 11 \mu \mathrm{m} \\
\text { diam }(\mathrm{mas})\end{array}$ & $\begin{array}{r}\text { diam } \\
(\mathrm{mas})\end{array}$ \\
\hline$o$ Ceti & $28.8^{1}$ & $25.6^{2}$ & $50^{3}$ & 69.8 \\
R Leonis & $30.7^{1}$ & $26.3^{3}$ & $70^{3}$ & 62.3 \\
W Hydrae & $39.9^{4}$ & & & 66.0 \\
U Herculis & $11.0^{1}$ & & & 20.8 \\
OH26.5+0.6 & & $\sim 7^{5}$ & $\sim 40^{5}$ & 20.8 \\
\hline
\end{tabular}

Notes: ${ }^{1}$ Mennesson et al. 2002; ${ }^{2}$ Weiner (2004); ${ }^{3}$ Danchi et al. (1994); ${ }^{4}$ Millan-Gabet et al. (2005); ${ }^{5}$ Model from Suh (2004).

Ireland et al. (2004) gives aperture masking results which show that the photosphere is not well modeled with a uniform disk and a Gaussian model is used instead. The size of the Gaussian FWHM varied systematically from about 40 mas at $0.68 \mu \mathrm{m}$ to 20 mas at $0.92 \mu \mathrm{m}$ with significantly larger sizes seen in the TiO bands (phase 0.34).

Interferometric measurements at $2.2 \mu \mathrm{m}$ using the VLTI/ VINCI instrument are reported by Woodruff et al. (2004) who show the diameter varying between 29 and 35 mas. Measurements using several narrow bands near 2.2 and $3.6 \mu \mathrm{m}$ using the IOTA/FLUOR interferometer lead Perrin et al. (2004) to conclude that the star had a molecular layer in which opacity due to $\mathrm{H}_{2} \mathrm{O}$ caused the apparent variation of size with wavelength. The photospheric diameter reported was 26.8 mas in 2000 (phase 0.01) and 25.0 mas in 2001 (phase 0.20).

Danchi et al. (1994) reports $11 \mu \mathrm{m}$ observations to which a photospheric diameter varying between 38.6 and 47.2 mas and an inner diameter of the dust shell of 120 mas was fit. Subsequent measurements with the ISI at $11 \mu \mathrm{m}$ by Weiner et al. (2003b) from 1999 to 2001 gave a diameter varying between 42.6 and 48 mas and some evidence for an asymmetric structure. Later, modeling by Weiner (2004) concluded that the $11 \mu \mathrm{m}$ data could be explained by a photospheric diameter of 25.6 mas surrounded by a shell of $\mathrm{H}_{2} \mathrm{O}$ with a diameter of 60.5 mas. Atmospheric modeling by Ohnaka et al. (2005) concluded that both interferometric and spectroscopic observations of $o$ Ceti could largely be explained by an $\mathrm{H}_{2} \mathrm{O}$ layer surrounding the star. Modeling of the SED of $o$ Ceti was reported by Suh (2004) who claimed that a $10 \mu \mathrm{m}$ opacity of only $0.01-0.02$ could account for the observed SED. This modeling concluded that the inner diameter 

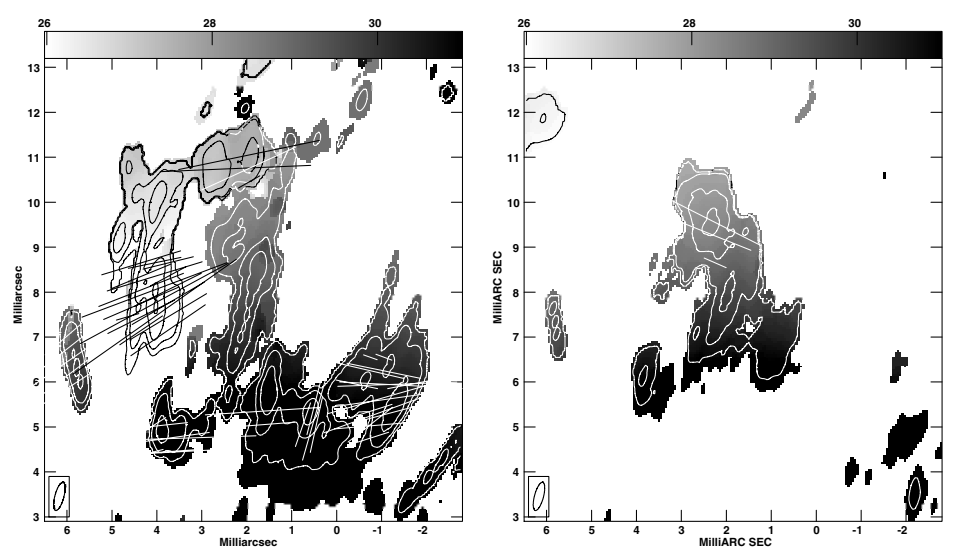

Fig. 9. $\mathrm{OH} 26.5+0.6$. Closeup of a region in Fig. 5. Left is the $v=2, J=1-0$ transition of $\mathrm{SiO}$ at $42.8 \mathrm{GHz}$, on the right is the $v=1, J=$ 1-0 transition of $\mathrm{SiO}$ at $43.1 \mathrm{GHz}$. The gray scale is the average velocity as given by the scale-bar at the top, contours are total intensity with an interval of powers of two, and polarization "E" vectors are over-plotted. The center of the star is below. The resolution is shown in a box in the lower left corner.

of the dust shell was 27 to 30 times the diameter of the star or considerably larger than observed by Danchi et al. (1994).

McIntosh (2006) reports on frequent spectroscopic monitoring of several of the $\mathrm{SiO}$ masers in $o$ Ceti. The $v=1, J=$ 1-0 transition showed a correlation of velocity with integrated flux density over the pulsation cycle. Interferometric monitoring of $\mathrm{SiO}$ masers during 2001 are reported by Cotton et al. (2004); the ring diameter varied between 60 and 75 mas. Cotton et al. (2006) report observations at an additional four epochs in 2003 and 2004 with $\mathrm{SiO}$ maser ring diameters varying from 60 to 80 mas.

The diameter of the the $\mathrm{SiO}$ ring given in Table 2, 69.8 mas, is within the range previously reported by Cotton et al. (2004) and Cotton et al. (2006) and is somewhat larger than the 50 mas inner diameter of the dust shell reported by Danchi et al. (1994). This is consistent with the $\mathrm{SiO}$ masers marking the region just interior to the region of silicate dust formation.

Figures 1 and 6 show systematic velocity variations along numerous radial features. In these features, the observed radial velocities are furthest from systemic closest to the photosphere. Since the line of sight is tangent to the maser ring, these motions are tangential as seen by the star and so are not explained by simple radial accelerations. The predominantly perpendicular polarization vectors seen in Fig. 6 suggest that these motions are controlled by the magnetic field of the star.

It is possible that the radial features seen in Figs. 1 and 6 are the result of a mass ejection associated with the X-ray burst a year earlier reported by Karovska et al. (2005). At the Hipparcos (ESA 1997) distance of $128 \mathrm{pc}$, the average projected velocity of material ejected in the flare reaching the position of the linear feature seen in Fig. 6 is about $13 \mathrm{~km} \mathrm{~s}^{-1}$. This value is somewhat larger than the total range of maser velocities observed in the star but higher than average velocities for ejected material closer to the stellar surface are likely.

Similar linear features were prominent during only one of the four epochs during 2001 sampled by Cotton et al. (2004). This leads to the possibility that stellar flaring activity may contribute to the overall mass loss process.

\section{2. $R$ Leonis}

Masked aperture interferometry by Ireland et al. (2004) between 0.68 and $0.92 \mu \mathrm{m}$ show an increasing size towards the blue going from 22 to 40 mas (phase 0.91). Non-zero closure phases indicate asymmetries in the structure. Monnier et al. (2004) used a combination of aperature masking on Keck with interferometric measurements using IOTA at $2.2 \mu \mathrm{m}$ to image $\mathrm{R}$ Leo. A uniform disk of diameter 30.3 mas (phase 0.90 ) adequately explained the data. R Leo was observed by Fedele et al. (2005) at $2.2 \mu \mathrm{m}$ using VLTI/VINCI who derived diameters of 28.5 and 26.2 mas at successive maxima. The shape of the visibility curve was consistent with a circumstellar molecular envelope. Millan-Gabet et al. (2005) give $H$ and $K$ band apparent sizes of 23.8 and 29.9 mas measured with IOTA (phase 0.4). Schuller et al. (2004) attempted modeling observations of R Leo and concluded that a model with only a photosphere and a dust shell could account for observations at 2.2 and $11 \mu \mathrm{m}$ but not $3.6 \mu \mathrm{m}$. Using several narrow band observations between 2.2 and $3.6 \mu \mathrm{m}$, Perrin et al. (2004) fitted a model of a photospheric diameter of 21.8 mas surrounded by an $\mathrm{H}_{2} \mathrm{O}$ layer 50 mas in diameter in 2000-2001.

Weiner et al. (2003a) report measurements on $\mathrm{R}$ Leo at $11 \mu \mathrm{m}$ with ISI measured apparent sizes between 55.3 and 64.2 mas in the period 1999-2001. ISI observations at $11 \mu \mathrm{m}$ by Tatebe et al. (2006) show dramatic changes in the star to dust ratio between 2003 and 2004 (less dust in 2004) but zero closure phases suggest symmetric structure.

Cotton et al. (2004) observed R Leo at four epochs during 2001 and reported variations of the $\mathrm{SiO}$ ring diameter of 50 to 61 mas. At none of these epochs was anything like the striking set of linear features shown in Figs. 2 and 7 seen. The diameter of the $\mathrm{SiO}$ ring given in Table 3, 62.3 mas is at the upper end of the range of values seen in 2001 and is slightly smaller than the inner diameter of the dust shell, 70 mas, from Danchi et al. (1994). This size is slightly larger that the estimated size of the molecular layer, 50 mas, estimated by Perrin et al. (2004) (phase 0.80).

\subsection{W Hydrae}

On the basis of modeling of ISO spectroscopy, Justtanont et al. (2004) argue for a warm $\mathrm{CO}_{2}$ layer between the photosphere and the dust shell. Masked aperture interferometry by Ireland et al. (2004) between 0.68 and $0.92 \mu \mathrm{m}$ show an increasing size towards the blue going from 40 to 70 mas (phase 0.44 ) with some evidence of asymmetric structure. Monnier et al. (2004) used a combination of aperture masking on Keck with interferometric measurements using IOTA at $2.2 \mu \mathrm{m}$ to image W Hya. A uniform disk model (42.5 mas) gave a poor fit (phase 0.00). 
Millan-Gabet et al. (2005) give $H$ and $K$ band apparent sizes of 32.2 and 39.9 mas measured with IOTA (phase 0.6).

Cotton et al. (2004) observed W Hya in August 2001 and give an average $\mathrm{SiO}$ ring diameter of 80 mas which as considerably larger than the 66 mas given in Table 3.

\subsection{U Herculis}

Ragland et al. (2006) fit a uniform disk model at $H$ band with a diameter of 9.7 mas in Jun. 2002 using IOTA. A non-zero closure phase indicates asymmetric structure. Mennesson et al. (2002) give a uniform disk diameter of 11 mas at $2.2 \mu \mathrm{m}$ and 14.3 mas at $3.6 \mu \mathrm{m}$ in 2000 (phase 0.25 ). U Her was observed at four epochs in 2001 by Cotton et al. (2004) and had a maser ring diameter between 22 and 25 mas. The ring diameter given in Table 3 of 22.8 mas is within this range.

The character of the source is quite different as shown in Figs. 4 and 8 and in the 2001 images of Cotton et al. (2004); showing more extended emission and a more chaotic and less ring-like structure in the observations presented here. This is illustrated in the ring widths in Table 2 which are roughly twice the typical value in Cotton et al. (2004). Figure 8 shows linear radial features with systematic radial velocity variations; this type of feature was not observed during 2001. The large radial extent of the masing region suggests that its outer size in not being truncated by condensation of $\mathrm{SiO}$ onto grains.

\section{5. $\mathrm{OH} 26.5+0.6$}

Chesneau et al. (2005) reports on the MIDI observations of $\mathrm{OH} 26.5+0.6$. The star was resolved by the beams of the $8 \mathrm{~m}$ telescopes with a strong asymmetry and a size of $286 \times 214$ mas. No fringes were detected interferometrically. However, modeling of the SED by Suh (2004) predicts an opacity between 15 and 22 at $10 \mu \mathrm{m}$ which would obscure the central star. The lack of interferometric detection of the dust shell suggests that it is quite smooth. The modeling of Suh (2004) predicts that the inner diameter of the dust shell will be approximately 40 mas, or roughly twice the diameter of the $\mathrm{SiO}$ maser ring from Table 3 . The image of the $\mathrm{SiO}$ masers shown in Figs. 5 and 9 show a relatively chaotic structure with a very large width. This is consistent with the prediction of Suh (2004) that the maser ring is well inside the dust condensation radius and that the masers are not truncated on the outer edge by the $\mathrm{SiO}$ condensing onto grains.

\section{Conclusions}

We present new total intensity and linear polarization VLBA observations of the $v=2$ and $v=1 J=1-0$ maser transitions of $\mathrm{SiO}$ at 42.8 and $43.1 \mathrm{GHz}$ of a number of AGB stars being studied jointly with the VLBA and VLTI/MIDI interferometers.

The unusually large radial extent of masers around $\mathrm{U}$ Herculis and $\mathrm{OH} 26.5+0.6$ suggest that the outer regions of the maser ring are not being truncated by the condensation of the $\mathrm{SiO}$ onto dust grains. In the latter case, this is supported by the modeling of Suh (2004) suggesting that the region of dust condensation is well outside of the region in which masers are observed. If this conjecture is correct, then the stellar distance of dust formation is variable in U Herculis as earlier observations by Cotton et al. (2004) show a much narrower radial extent of the $\mathrm{SiO}$ masing region. These observations support the suggestion that the physical conditions in the outer envelopes of these stars are quite variable in time.
There is an intriguing possibility of an association of the X-ray flare in Mira reported by Karovska et al. (2005) with the linear features seen in the $\mathrm{SiO}$ maser images presented here. This suggests that flaring activity could play a role in the mass-loss process.

The polarization structure of the linear features also show a preference for the $\mathrm{E}$ vectors to be perpendicular to the direction of the feature; this is interpreted as indicating magnetic fields oriented primarily along the feature. Two possible explanations for this are the magnetic field being dragged by the ionized component of the envelope or the magnetic field controlling the motion of the accelerating material. Several recent $\mathrm{SiO}$ and $\mathrm{H}_{2} \mathrm{O}$ maser observation indicate that the magnetic field will be dynamically important (Herpin et al. 2003; Vlemmings et al. 2005, 2006). Thus, the jet-like structure are likely due to the masing regions being dragged along the magnetic field. Observations of such features sufficiently closely spaced in time to measure proper motions should help understand the nature of these features.

\section{References}

Chesneau, O., Verhoelst, T., Lopez, B., et al. 2005, A\&A, 435, 563 Cotton, W. D., Mennesson, B., Diamond, P. J., et al. 2004, A\&A, 414, 275 Cotton, W. D., Vlemmings, W., Mennesson, B., et al. 2006, A\&A, 456, 339 Danchi, W. C., Bester, M., Degiacomi, C. G., Greenhill, L. J., \& Townes, C. H. 1994, AJ, 107, 1469

Deguchi, S., \& Watson, W. D. 1990, ApJ, 354, 649

Diamond, P. J., \& Kemball, A. J. 2003, ApJ, 599, 1372

Diamond, P. J., Kemball, A. J., Junor, W., et al. 1994, ApJ, 430, L61

Elitzur, M. 1991, ApJ, 370, 407

Elitzur, M. 1996, ApJ, 457, 415

ESA 1997, VizieR Online Data Catalog, 1239, 0

Fedele, D., Wittkowski, M., Paresce, F., et al. 2005, A\&A, 431, 1019 Herpin, F., Baudry, A., Thum, C., Morris, D., \& Wiesemeyer, H. 2003, in SF2A2003: Semaine de l'Astrophysique Francaise, 523

Herpin, F., Baudry, A., Thum, C., Morris, D., \& Wiesemeyer, H. 2006, A\&A, 450,667

Humphreys, E. M. L., Gray, M. D., Yates, J. A., et al. 1996, MNRAS, 282, 1359 Humphreys, E. M. L., Gray, M. D., Yates, J. A., et al. 2002, A\&A, 386, 256 Ireland, M. J., \& Scholz, M. 2006, MNRAS, 367, 1585

Ireland, M. J., Tuthill, P. G., Bedding, T. R., Robertson, J. G., \& Jacob, A. P. 2004, MNRAS, 350, 365

Justtanont, K., de Jong, T., Tielens, A. G. G. M., Feuchtgruber, H., \& Waters, L. B. F. M. 2004, A\&A, 417, 625

Karovska, M., Schlegel, E., Hack, W., Raymond, J. C., \& Wood, B. E. 2005, ApJ, 623, L137

Kemball, A. J., \& Diamond, P. J. 1997, ApJ, 481, L111

McIntosh, G. C. 2006, ApJ, 649, 406

Mennesson, B., Perrin, G., Chagnon, G., et al. 2002, ApJ, 579, 446

Menut, J.-L., Chesneau, O., Lopez, B., et al. 2004, in New Frontiers in Stellar Interferometry, ed. W. A. Traub (Bellingham, WA: The International Society for Optical Engineering), Proc. SPIE, 5491, 1733

Millan-Gabet, R., Pedretti, E., Monnier, J. D., et al. 2005, ApJ, 620, 961

Monnier, J. D., Millan-Gabet, R., Tuthill, P. G., et al. 2004, ApJ, 605, 436

Nedoluha, G. E., \& Watson, W. D. 1990, ApJ, 354, 660

Nedoluha, G. E., \& Watson, W. D. 1994, ApJ, 423, 394

Ohnaka, K., Bergeat, J., Driebe, T., et al. 2005, A\&A, 429, 1057

Perrin, G., Ridgway, S. T., Mennesson, B., et al. 2004, A\&A, 426, 279

Ragland, S., Traub, W. A., Berger, J.-P., et al. 2006, ApJ, 652, 650

Reid, M. J., \& Menten, K. M. 1997, ApJ, 476, 327

Reid, M. J., \& Moran, J. M. 1981, ARA\&A, 19, 231

Schuller, P., Salomé, P., Perrin, G., et al. 2004, A\&A, 418, 151

Suh, K.-W. 2004, ApJ, 615, 485

Tatebe, K., Chandler, A. A., Hale, D. D. S., \& Townes, C. H. 2006, ApJ, 652, 666

Vlemmings, W. H. T., Diamond, P. J., \& Imai, H. 2006, Nature, 440, 58

Vlemmings, W. H. T., van Langevelde, H. J., \& Diamond, P. J. 2005, A\&A, 434, 1029

Weiner, J. 2004, ApJ, 611, L37

Weiner, J., Hale, D. D. S., \& Townes, C. H. 2003a, ApJ, 589, 976

Weiner, J., Hale, D. D. S., \& Townes, C. H. 2003b, ApJ, 588, 1064

Woodruff, H. C., Eberhardt, M., Driebe, T., et al. 2004, A\&A, 421, 703 